\title{
Dye-Laser Development for Plasma Magnetic-Field Diagnostic
}

Paul G. Weber

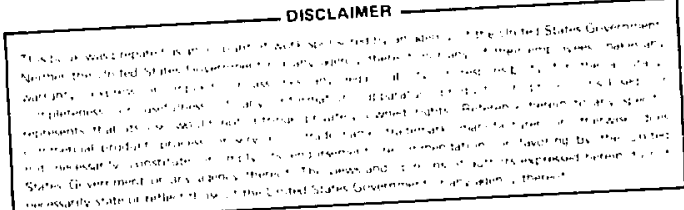




\title{
DYE LASER DEVELOPMENT FOR PLASMA MAGNETIC FIELD DIAGNOSTIC
}

by

\author{
Paul G. Weber
}

\begin{abstract}
A flash-lamp-pumped dye laser has been constructed and operated in DCM dye, yielding outputs greater than $400 \mathrm{~W}$ for $100 \mu \mathrm{s}$ in broadband operation. Attempts to tune this laser by injection locking to a narrow-band $\mathrm{cw}$ laser showed poor efficiency and relatively short locked operation.
\end{abstract}

\section{INTRODUCTION}

Measurement of confining magnetic fields in a hot plasma is not possitle using the cold plasma technique of inserting a material pickup loop. However, the magnetic field direction in a Tokamak plasma has been determined by McCormick, ${ }^{1}$ who injected a beam of lithium atoms into the discharge and observed the polarization of a lithium Zeeman component. Plasma electrons were responsible for the excitation of the lithium, leaving a substantial fraction of the lithium atoms unused in the ground state.

We have embarked on a more sophisticated version of this diagnostic, employing a higher current density and more energetic lithium beam, and utilizing dye laser resonant fluorescence techniques to detect the Zeeman pattern. ${ }^{2}$ In this scheme, the dye laser could be wavelength tuned, giving resonance only at a particular magnetic field strength. Alternatively, the laser polarization could be rotated, giving maximum fluorescence when the laser polarization direction coincides with the local magnetic field orientation. We have calculated that we require a tuned dye laser power of $7 \mathrm{~W}$ to saturate the $670.8-\mathrm{nm}$ lithium transition, with a bandwidth of 0.004 $\mathrm{nm}$ and pulst duration of at least $10 \mu$ s to permit the use of modulation techniques. We report here on the development of a high-power flash-lamp-pumped dye laser, and on attempts to tune this laser by injection locking to a cw dye laser.

\section{EXPERIMENTAL S YSTEM}

The dye laser head is modified from a Korad K-1 ruby laser system by replacing the ruby rod with an appropriate Pyrex tube (Fig. 1). The inside diameter in the pumped region was $7 \mathrm{~mm}$ for the untuned, long-pulse laser system and $2 \mathrm{~mm}$ for the injection locking experiments. DCM dye ${ }^{3}$ at a concentration of $5 \times 10^{-5} \mathrm{M} / \ell$ in methyl alcohol was circulated through the system until 2 min before a pulse, when the laser head was isolated. An $18-\mathrm{kV}, 1-\mu \mathrm{s}$ prepulse ${ }^{4}$ was applied to the flash lamp immediately before discharge of the lumped-element transmission line shown in Fig. 2. The capacitors were usually charged to $12-17 \mathrm{kV}$, giving a flat-topped current pulse of 3-4 kA. Various mirrors were used; lasing was achieved for reflectivity combinations from $40 / 99 \%$ to 99/99\%. To obtain long-pulse lasing in DCM dye, we found it essential to bubble dry nitrogen gas through the dye to act as a triplet quencher. Figure 3 shows the 


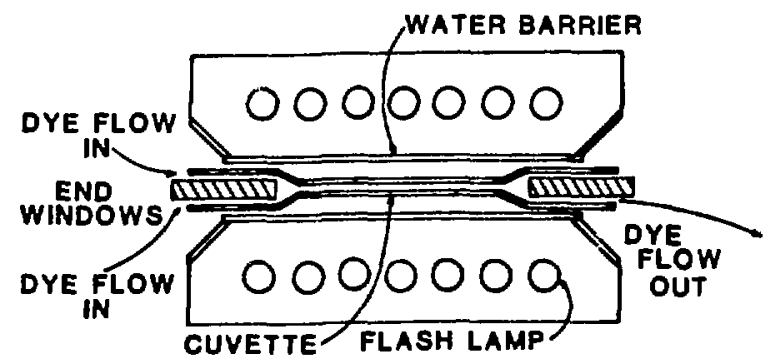

Fig. I. Detail of ruby laser head, modified for dye operation.

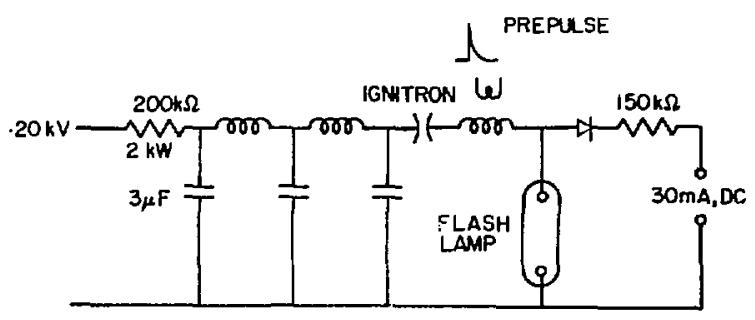

Fig. 2. Electrical circuit for hash lamp.
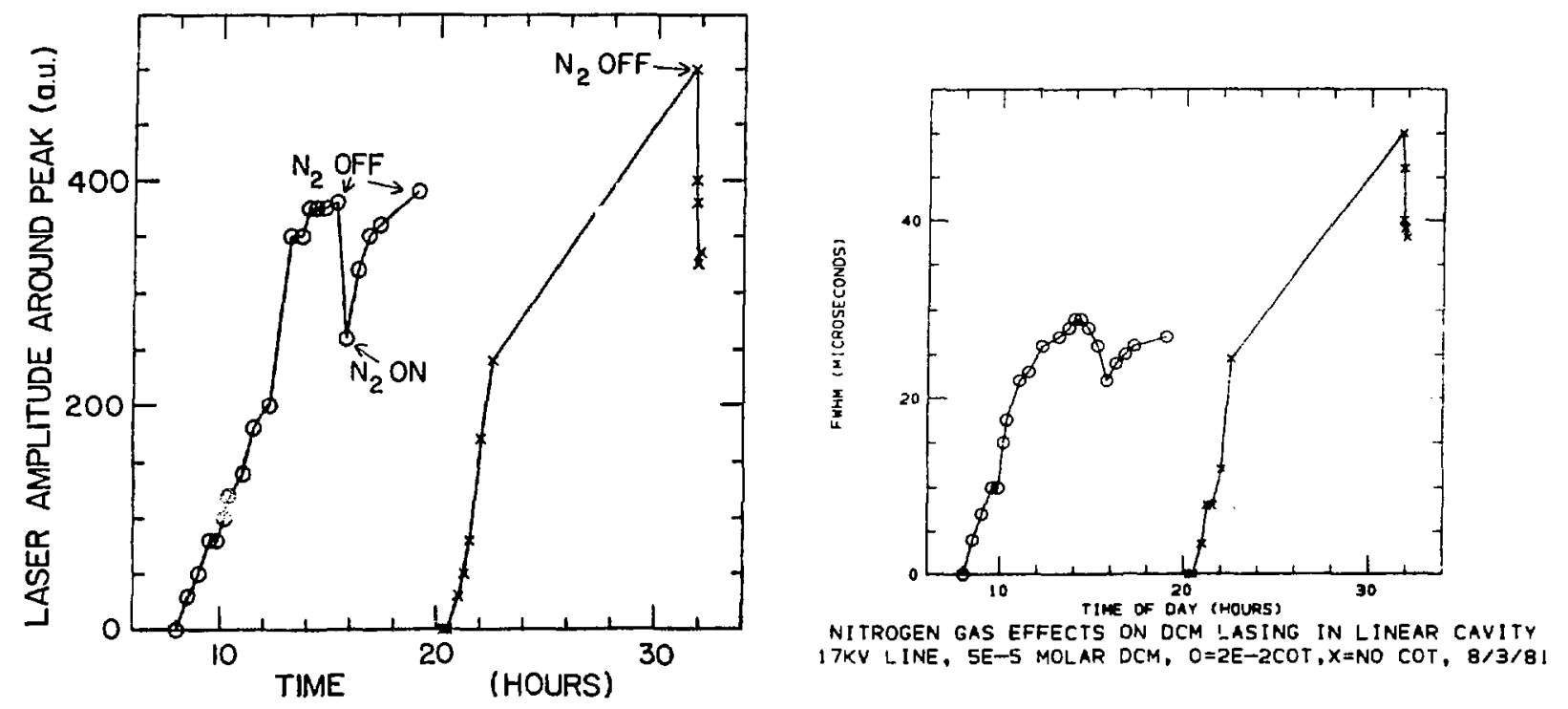

Fig. 3. Effect of nitrogen gas as a triplet quencher for DCM dye.

cumulative effect of nitrogen gas on laser power and pulse length. The addition of the traditional triplet quencher cyclooctatetraene (COT) had no appreciable effect on laser operation.

\section{BROADBAND OPERATION}

The total output of the laser was measured using a calibrated photodiode, fitted with a high-pass cutoff filter to discriminate against flash-lamp light. Peak powers of $1 \mathrm{~kW}$ were measured for pulses of $50-\mu \mathrm{s}$ FWHM, with emission exceeding $400 \mathrm{~W}$ to $100 \mu \mathrm{s}$. A typical output signal is shown in Fig. 4. Note the spiked nature of the signal, which sugges $;$ that the laser emission is rapidly changing.

Initial spectral analysis of this output was performed by dispersing the light in a $0.5-\mathrm{m}$ Jarrell-Ash spectrometer, whose exit slit had beet replaced by a Reticon self-scanned photodiode array. After clearing the diode array, the flash lamp was triggered, and the array was read immediately following current termination. The information obtained is the time integrated spectral emission during the flash-lamp pulse in a quantitative format. We found that the laser operates over an $11-\mathrm{nm}$ wide bandwidth centered at $644 \mathrm{~nm}$. This center wavelength is near that observed in our $\mathrm{cw}$ dye laser when operated with DCM dye. 


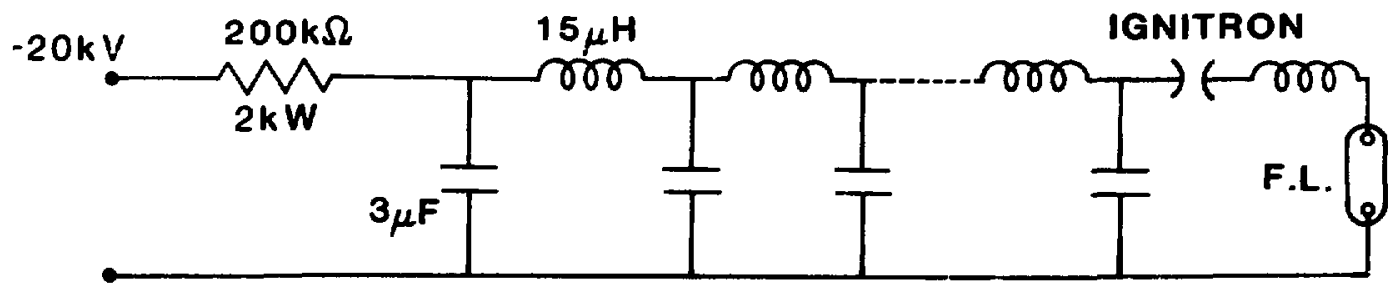

\title{
FLASHLAMP PUMPING CIRCUIT
}

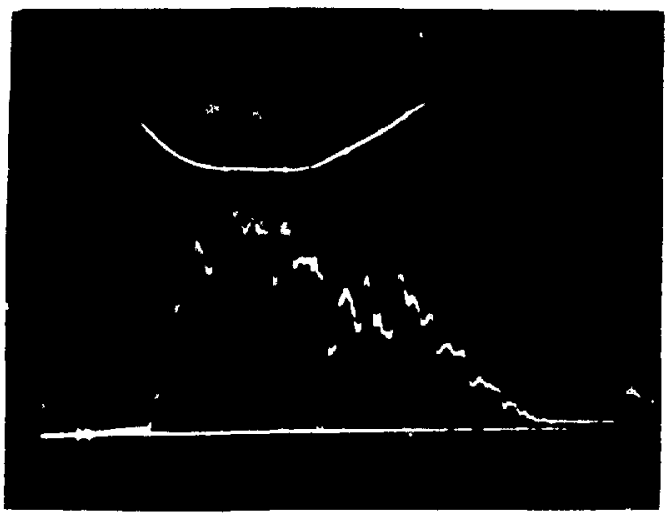

\author{
FLASHLAMP CURRENT, $1 \mathrm{kA}$ /DIV.
}

LASER OUTPUT, 100W/DIV.

$20 \mu s / D I V$.

\section{LINEAR CAVITY PERFORMANCE DCM DYE, NO INJECTION.}

Fig. 4. Typical broadband performance of dye laser.

\section{INJECTION LOCKING}

Two observations of the spatial output characteristics of the untuned pulsed laser forced modifications before the injection locking experiments. (1) With a 7 -mm-i.d. dye tube, most absorption of pump light by the dye was in the outer $2 \mathrm{~mm}$, giving a "donut" mode when lasing. More uniform output was obtained by using a 2-mm-i.d. dye tube in the locking experiments. (2) A cw laser beam passing along the dye tube axis was deflected during the flash-lamp pulse due to thermal gradients developing in the dye. (A similar effect has been noted in dye amplifier cells. ${ }^{5}$ ) To minimize dye heating before reaching threshold, we reduced the external system inductance and applied a simmer power supply to the flash lamp. ${ }^{6}$ This reduced the discharge inductance of the flash lamp by a factor of two and reduced the delay from current initiation to laser threshold from 10 to $7 \mu \mathrm{s}$. We also sharply reduced the length of the current pulse to prolong flash-lamp life.

All injection experiments were performed near the peak wavelength of free lasing, which is $644 \mathrm{~nm}$. The pulsed laser cavity length was $0.54 \mathrm{~m}$, with fine adjustment provided by mounting one mirror on a piezoelectric translator. Longitudinal mode matching was achieved using the theory of Kogelnik. ${ }^{7}$ Two different sets of mirrors were used, with qualitatively the same locking properties being achieved. We discuss here the results obtained with a $60 \%$ reflecting input coupler and a $99 \%$ output mirror. The injection signal was provided by a Coherent $595 \mathrm{cw}$ dye laser operating in DCM dye with a bandwidth of $3 \mathrm{GHz}$. Injection powers were measured at the $\mathrm{cw}$ laser output and a range up to $75 \mathrm{~mW}$. The pulsed laser output was attenuated, focused onto the 20 $\mu \mathrm{m}$-wide entrance slit of the $0.5-\mathrm{m}$ spectrometer, and detected by an IP 21 photomultiplier tube. Typically, in broadband operation, $4 \mathrm{~W}$ of power were measured in a $\leq 0.1-\mathrm{nm}$ observation window looking at the $99 \%$ reflector. Proportionally higher powers were seen with a less reflecting output coupler.

The following observations are made concerning the first several microseconds of lasing.

(1) The output power at the injected wavelength increases linearly with injected power, reaching four times the broadband value with $30 \mathrm{~mW}$ of $\mathrm{cw}$ power.

(2) Laser power at wavelengths near that of the injection are reduced by up to $30 \%$ under these conditions. 
(3) Fine tuning of mode matching using the piezoelectrically adjusted laser mount improves locking efficiency by up to $50 \%$.

(4) Injection locking is effective only for a few microseconds.

(5) Locking efficiency is not noticeably affected by the power of the pulsed laser, although the locked time decreases at higher pumping power.

The short duration of the locked pulse is attributed to thermally induced refractive index changes in the dye during the pulse. These index changes alter the effective length of the cavity and induce a frequency "chirp" in the laser. ${ }^{B}$ Indeed, for a typical $500-\mathrm{J}$ capacitor energy deposited into the laser head, in a $40-\mu$ s pulse, we see a $1.2^{\circ} \mathrm{C}$ rise in dye temperature. Assuming a uniform heating rate, this translates to a cavity length change of order $1 \mu \mathrm{m} / \mu \mathrm{s}$. This shift is sufficient to decrease the locking efficiency at the original wavelength to a small enough value for locking to fail almost immediately. The observation of shorter locked pulses at higher energy inputs supports this explanation.

\section{Y. CONCLUSION}

We have built and successfully operated a flash-lamppumped dye laser in DCM dye with pulse duration of up to $100 \mu$ s and peak power of $1 \mathrm{~kW}$. The laser output is spatially nonuniform, and the emission wavelength changes rapidly during the shot.

A number of modifications were made to allow an examination of injection locking in a long-pulsed dye laser. It was found, due to the high-energy density in the laser head, that thermal refractive index changes were taking place during the pulse. This caused frequency shifts in the laser and co sequent failure of the locking. Because high power, narrow band, long pulses are diff,cult to obtain from this sytem, we are considering using a train of cavity dumped pulses for resonance fluorescence detection of lithium in the magnetic field diagnostic.

\section{ACKNOWLEDGMENTS}

The initial part of this research was guided by the work of Peter R. Forman. I also wish to thank Randall M. Erickson, who implemented the first design, and Terry Langham for technical assistance.

\section{REFERENCES}

I. K. McCormick and J. Olivain, Rev. Phys. Appl. 13, $85(1: 78)$.

2. P. G. Weber and R. M. Erickson, Bull. Am. Phys. Soc. 26, 966 (1981).

3. Laser Dyes, Exciton Chemical Company, Inc., Dayton, Ohio (1981).

4. M. H. Ornstein and V. E. Derr, Appl. Opt. 13, 2100 (1974).

5. T. W. Hansch, F. Varsanyi, and A. L. Schanlow, Appl. Phys. Lett. 18, 108 (1971).

6. A. Hirsh, R. Meyer, and K. Schetter, Opt. Commun. 40, 63 (1981).

7. H. Kogelnik, Bell Syst. Tech. J., 334 (1964).

8. A. A. Pease, O. G. Peterson, M. L. Spaeth, and W. M. Pearson, IEEE J. Quant. El. QE 13, 280 (1977). 